\title{
Cálculo de parámetros eléctricos en líneas de transmisión de alta tensión
}

\section{Calculation of electrical parameters in high voltage transmission lines}

ALBINO-PADILLA, Ismael $\uparrow *$, ESCAMILLA-SANCHEZ, Juan Carlos, MORALES-AGUILAR, Eric y MARTINEZ, Daniel F.

\author{
Benemérita Universidad Autónoma de Puebla \\ Universidad de Colima \\ ID $1^{\text {st }}$ Autor: Ismael, Albino-Padilla \\ ID $1^{\text {er }}$ Coautor: Juan Carlos, Escamilla-Sánchez / CVU CONACYT ID: 230175 \\ ID $2^{\text {do }}$ Coautor: Eric, Morales-Aguilar / CVU CONACYT ID: 225102 \\ ID $3^{\text {er }}$ Coautor: Daniel F., Martínez
}

DOI: $10.35429 / J E A .2020 .22 .7 .1 .7$

Recibido: 10 de Enero, 2020; Aceptado: 29 de Marzo, 2020

\section{Resumen}

Este articulo tiene como finalidad determinar los parámetros electricos de las líneas de transmisión, se enfocará en el cálculo de la Inductancia y Capacitancia geométricas. Para esto se estudian los casos de las líneas monofásica y trifásica con espaciamiento equilátero. Se utiliza el programa de COMSOL Multiphysics para obtener los parámetros de las líneas. Los resultados de simulación se comparan conl as ecuaciones basicas clásicas.

Modelado del transformador, Transformada Numérica de Laplace, transitorios electromagnético

\begin{abstract}
This article aims to determine the electrical parameters of the transmission lines, it will focus on the calculation of geometric inductance and capacitance. For this, the cases of the single-phase and three-phase lines with equilateral spacing are studied. The COMSOL Multiphysics program is used to obtain the parameters of the lines. The simulation results are compared with the classic basic equations.
\end{abstract}

Lìneas de transmisión, COMSOL- Multiphysics Inductancia y Capacitancia Geometrica

Citación: ALBINO-PADILLA, Ismael, ESCAMILLA-SANCHEZ, Juan Carlos, MORALES-AGUILAR, Eric y MARTINEZ, Daniel F. Cálculo de parámetros eléctricos en líneas de transmisión de alta tensión. Revista de Aplicaciones de la Ingeniería. 2020. 7-22: 1-7.

\footnotetext{
* Correspondencia del Autor (Correo Electrónico: escamilla_14@hotmail.com)

$\dagger$ Investigador contribuyendo como primer autor.
} 


\section{Introducción}

COMSOL Multiphysics 3.4 es un paquete de simulación que facilita la construcción y arranque de simulaciones de circuitos, utiliza el método de elemento finito para estudiar procesos físicos que se puedan describir mediante ecuaciones en derivadas parciales (EDP). Está provisto de algoritmos de solución que pueden manejar problemas complejos de forma rápida y precisa, recientemente se ha mejorado la plataforma de interacción con el usuario lo que ha mejorado su facilidad de uso y flexibilidad. Con COMSOL Multiphysics se puede modelar prácticamente cualquier fenómeno a través de plantillas de predefinidas. (COMSOL Multiphysics, 2015). Para realizar las simulaciones en este programa se hace uso de COMSOL Multiphysic en el módulo AC/DC, utilizando la librería Magnetostatics (Perpendicular Induction Currents) para el cálculo de inductancia y Electrostatics para el cálculo de la capacitancia (COMSOL Multiphysics, 2006).

\section{Cálculo de la inductancia de una línea monofásica}

La línea de transmisión monofásica en consideración, contiene dos conductores uno de los cuales lleva la corriente y el otro es el retorno, esta configuración es estudiada exhaustivamente en, también existe la configuración de la línea monofásica con retorno por tierra, la cual es usada ampliamente es zonas rurales, en el caso de estudio la línea monofásica de la Fig. 1, consiste de 2 conductores sólidos de radio $r_{1}=r_{2}=0.01 \mathrm{~m}$, separados una distancia $D=1 \mathrm{~m}$. Los conductores llevan corriente eléctrica de igual magnitud, pero en direcciones opuestas. Estas corrientes generan un campo magnético que enlaza a los conductores, este arreglo se conoce como línea a par abierto. (Morched, Gustavsen, \& Tartibi, 1999); (Gustavsen \& Adam, 1988); (Moreno, Gomez, Naredo, \& J. L. Guardado, 2005).
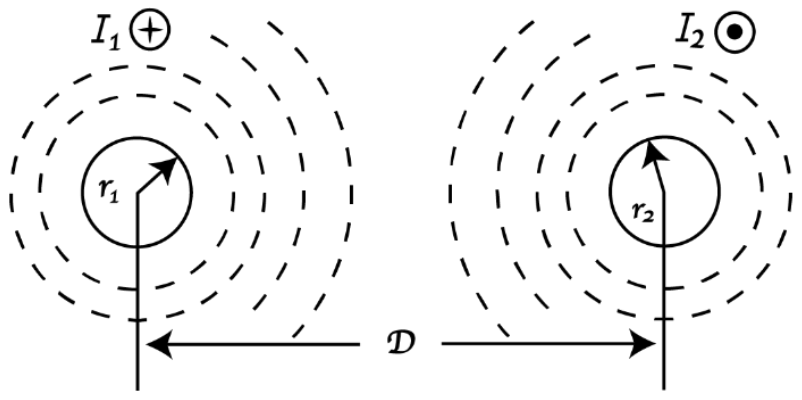

Figura 1 Línea de transmisión monofásica

La inductancia de la línea se puede calcular a partir de la siguiente expresión (Grainger, 1996).

$$
\begin{aligned}
& L_{1}=2 * 10^{-7} \ln \left(\frac{D}{r^{\prime}}\right)[H / m] \\
& r^{\prime}=r_{1} * e^{-\frac{1}{4}}
\end{aligned}
$$

Sustituyendo los datos de la línea en (1) se obtiene.

$$
L_{1 \text { Teoria }}=0.97103\left[\frac{\mathrm{mH}}{\mathrm{km}}\right]
$$

Este valor servirá como punto de comparación con el resultado que se obtenga con el programa de elemento finito. Para poder obtener la inductancia de una línea monofásica en COMSOL Multiphysics se necesita dibujar la geometría y definir el área de solución, esta se define con ayuda de una frontera externa, esta frontera puede distorsionar el comportamiento de los campos magnético y eléctrico y causar errores en la solución, para evitar esto, la frontera externa se debe colocar a una distancia tal que $D e>>D$, donde $D e$ es la distancia o radio del centro del arreglo hacia la frontera externa, después se inyectan corrientes en cada uno de los conductores, para este caso se inyectaron corrientes de CD (Corriente Directa) en ambos conductores (D. Glover, 2008). Los resultados se muestran en la Figura 2. 


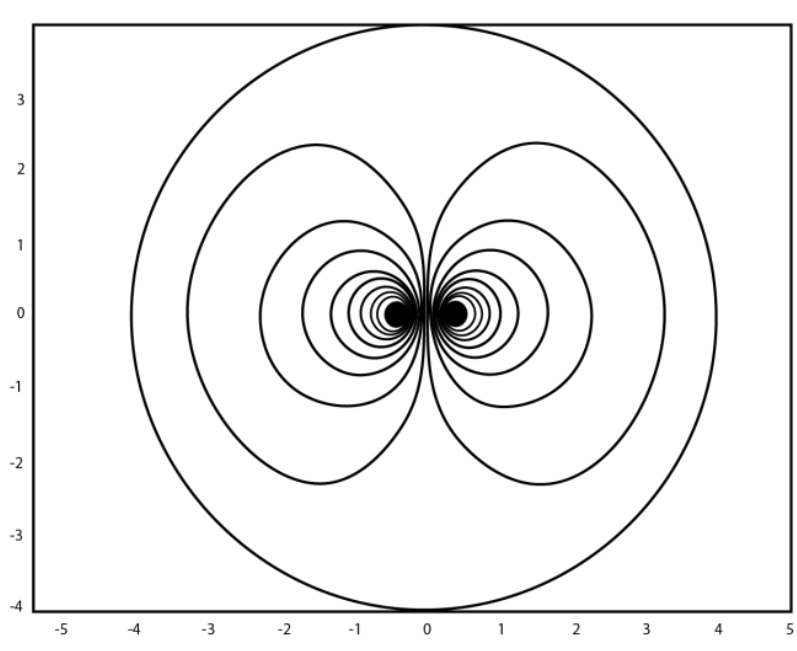

Figura 2 Distribución del campo magnético en la configuración monofásica de la línea simulación es

El flujo que se obtiene por medio de la $\psi($ flujo 1$)=1.93 * 10^{-6} \mathrm{~Wb}-$ Vuelta

Para un solo conductor tenemos que:

$\psi($ flujo 1$)=0.9610^{-6} \mathrm{~Wb}-$ Vuelta

La inductancia se obtiene usando la relación entre el flujo y la corriente. En la simulación se utilizó una corriente de excitación de 1A.

$L_{1 \operatorname{sim}}=\frac{\psi}{I}$

Sustituyendo los datos de la línea en (2) se obtiene.

$$
L_{1 \operatorname{Sim}}=0.965\left[\frac{\mathrm{mH}}{\mathrm{km}}\right]
$$

\section{Cálculo de la capacitancia de una línea monofásica}

Los conductores de la línea presentan una capacitancia entre ellos debido al gradiente de potencial. Esta capacitancia junto con la conductancia forma la admitancia de una línea. Para líneas aéreas suele despreciarse la conductancia ya que se considera el aire como dieléctrico perfecto (Clayton, 1994). La capacitancia es proporcional a la longitud de la línea y puede ser despreciada para una línea elecricamente cortas, para estudios de estado estable.
La capacitancia al neutro de una línea monofásica de la Fig. 3 se calcula mediante la siguiente ecuación, si $r_{1}=r_{2}=r$, tomando en cuenta que $r$ es el radio exterior del conductor no el RMG (Radio Medio Geométrico).

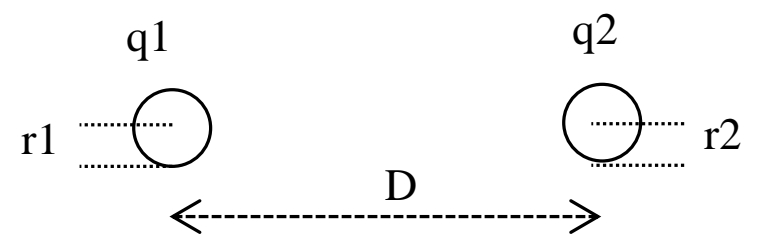

Figura 3 Línea monofásica usada para el cálculo de la capacitancia

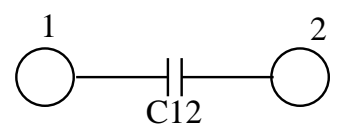

(a) Capacitancia LìneaLìnea

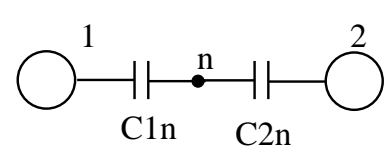

(b) Capacitancia LíneaNeutro
Figura 4 La capacitancia al neutro de una línea monofásica

$C_{1 n \text { Teoria }}=\frac{2 \pi \varepsilon}{\ln \frac{D}{r}}$

Donde:

$\varepsilon=8.854 * 10^{-12}\left[\frac{F}{m}\right]$

Sustituyendo datos de la configuración de la línea en (3) se obtiene que:

$C_{1 \text { Teoria }}=1.2080 * 10^{-11}\left[\frac{F}{m}\right]$

Para el cálculo de la capacitancia de la línea usando la simulación, al igual que en la inductancia se necesita dibujar la geometría de la línea, pero en este caso se aplican cargas de prueba en la superficie de los conductores, una carga positiva $+Q$ y una negativa $-Q$, en nuestro caso se decidió utilizar una carga de prueba de $Q=5 * 10^{-12} \mathrm{C}$. La distribución del campo eléctrico se muestra en la Fig. 5. Se utiliza las siguientes ecuaciones para encontrar la capacitancia entre conductores $C_{12} \quad \mathrm{y}$ al neutro $C_{1 n}$ de la Figura 4:

$C_{12}=\frac{Q^{2}}{2 w_{e}}$

$C_{1 n}=\frac{Q^{2}}{w_{e}}$ 
Donde:

$C_{12}=$ Capacitancia entre conductores

$C_{1 n}=$ Capacitancia al neutro

$Q=$ Carga de Prueba (C)

$w_{e}=$ Energia en el campo eléctrico $(\mathrm{J})$

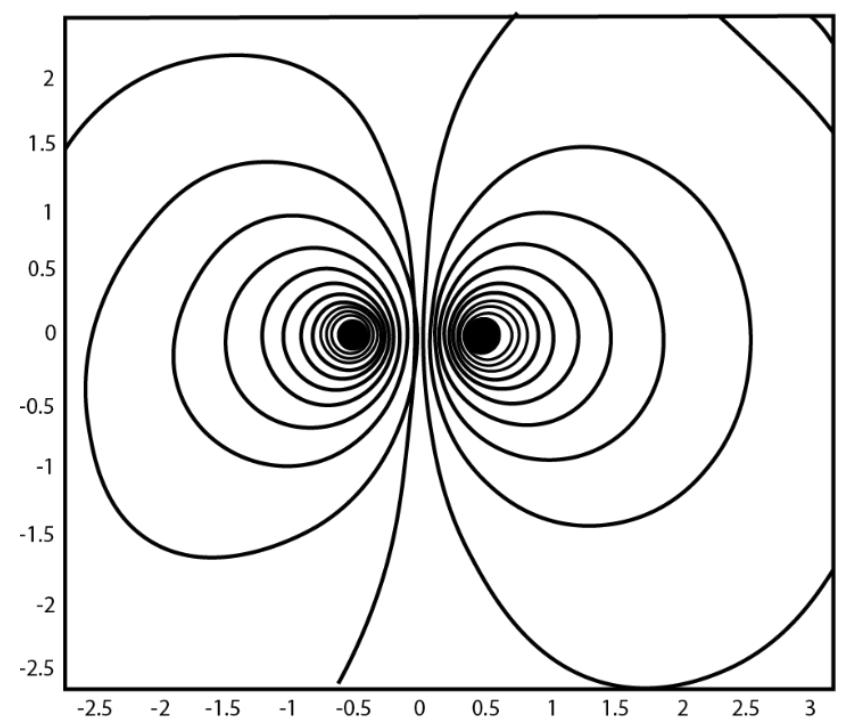

Figura 5 Distribución del campo eléctrico

De la simulación se obtiene, que:

$$
\mathrm{w}_{\mathrm{e}}=2.055296 * 10^{-12}[\mathrm{~J}]
$$

Sustituyendo el valor de $Q$ y $w_{e}$ en (5) se obtiene:

$$
\begin{aligned}
& C_{1 n}=\frac{\left(5 * 10^{-12}\right)^{2}}{2.055296 * 10^{-12}}\left[\frac{F}{m}\right] \\
& C_{1 \text { simulacion }}=1.216369 * 10^{-11}\left[\frac{F}{m}\right]
\end{aligned}
$$

\section{Línea de Transmisión trifásica con espaciamiento equilátero}

Como segundo caso se estudia la línea de transmisión trifásica, la cual tiene 3 conductores ACSR espaciados de manera equilátera como se muestra en la Fig. 6, con separación entre conductores de $0.836 \mathrm{~m}$.

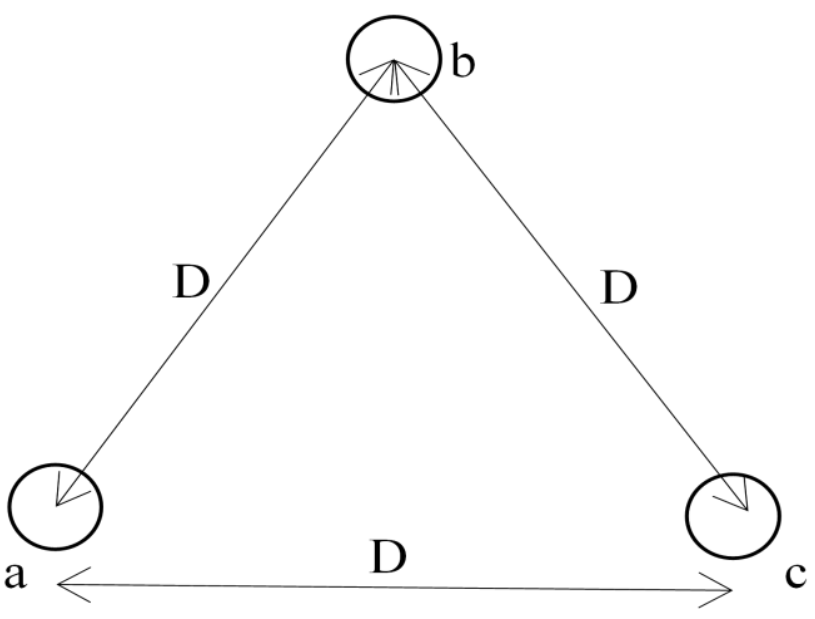

Figura 6 Configuración de la línea de transmisión trifásica

Para realizar el modelado necesitamos conocer los datos más importantes de la línea tales como su radio exterior y la distancia entre conductores, tomando en cuenta que la unidad de medida que se utilizará es el metro se realiza la conversión entre el sistema inglés y el internacional cuando sea necesario. Los datos característicos del conductor se obtuvieron haciendo uso de tablas "Electrical Characteristics of Bare Aluminum Conductor Steel-reinforced" (Grainger, 1996), de las cuales se obtienen los siguientes datos del conductor: Radio exterior de $0.01177 \mathrm{~m}$ Radio Medio Geométrico (RMG) de $0.00957 \mathrm{~m}$. La ecuación para determinar la inductancia de la línea trifásica con espaciamiento equilátero tiene cierto parecido a la de una línea monofásica. Debido a la simetría del arreglo, las inductancias de los conductores $b$ y $c$ son iguales a la de $a$. Como cada fase consiste en un solo conductor, la ecuación que se muestra determina la inductancia por fase de la línea trifásica:

$L_{a}=2 * 10^{-7} \ln \left(\frac{D}{D s}\right) \quad\left[\frac{H}{m}\right]$

Sustituyendo los datos de la geometría bajo estudio en (6)

$$
\begin{aligned}
& L_{\text {aTeoria }}=2 * 10^{-7} \ln \left(\frac{9}{0.0314}\right) \quad\left[\frac{H}{m}\right] \\
& L_{\text {aTeoria }}=1.1316 * 10^{-6} \quad\left[\frac{H}{m}\right]
\end{aligned}
$$

Obteniendo la inductancia trifásica tenemos:

$$
L_{3 \varphi}=3.3949 * 10^{-6} \quad\left[\frac{H}{m}\right]
$$


La obtención de la inductancia mediante simulación se puede simplificar si se considera la simetría de la geometría de la línea, así como una distancia de la frontera externa $D_{e}$ mucho mayor que la distancia entre los dos conductores $D$, es decir que la frontera debe estar a una distancia $D_{e}>D$ Realizando la simulación de la línea de transmisión trifásica como si fuera una línea monofásica, tomando en cuenta que el conducto b es usado como el retorno o también llamado neutro. La distribución del Campo magnético se muestra en la Fig. 7. El flujo total de la línea usando COMSOL entrega.

$\psi($ flujo $a)=2.268 * 10^{-6} \mathrm{~Wb}-$ Vuelta

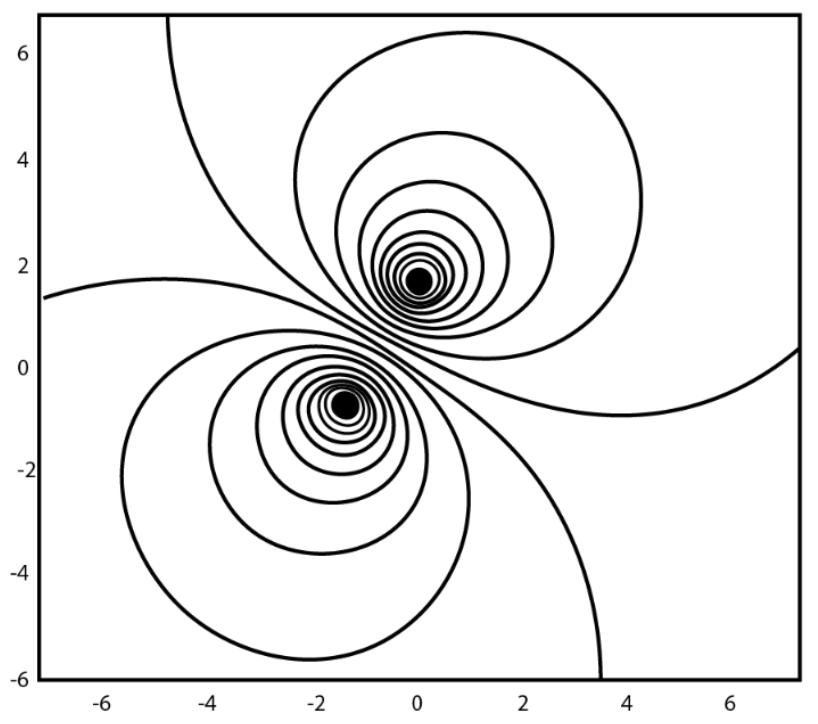

Figura 7 Distribución del campo magnético

Para un solo una fase tenemos que:

$\psi_{a}($ flujo $a)=1.134 * 10^{-6} \mathrm{~Wb}-$ Vuelta

Por tanto, para obtener su inductancia tenemos que:

$L_{a}=\frac{\psi}{I}=1.134 * 10^{-6}\left[\frac{H}{m}\right]$

La corriente de excitación se elige como 1A. Como siguiente paso ahora se obtiene la capacitancia de la línea trifásica, la capacitancia es el resultado de la diferencia de potencial entre conductores y origina que ellos se carguen de la misma forma que las placas de un capacitor cuando hay una diferencia de potencial.
La capcitancia representa el efecto del campo eléctrico con respecto al voltaje, su cálculo es de gran importancia ya que permite cuantificar la potencia reactiva que se inyecta a la línea la cual afecta directamente el voltaje en ambos extremos de la línea. Como la capacitancia al neutro es la relación de la carga sobre un conductor al voltaje entre el conductor y el neutro, tal parámetro puede calcularse como (D. Glover, 2008); (Grainger, 1996):

$C_{1 n T e o r i a}=\frac{2 \pi \varepsilon}{\ln \frac{D}{r}}$

Donde:

$\varepsilon=8.854 * 10^{-12}\left[\frac{F}{m}\right]$

Sustituyendo datos la configuración bajo estudio en (3).

$$
C_{1 n \text { Teoria }}=1.134934 * 10^{-11}\left[\frac{F}{m}\right]
$$

Ahora se procede a determinar el valor de la capacitancia por simulación, para reducir el problema y poder obtener el valor idóneo de la capacitancia en COMSOL, se consideró un conductor neutro equidistante de los tres conductores de fase esto de acuerdo con el diagrama fasorial de voltajes balanceados de una línea trifásica, Fig. 8 y Fig. 9.

$C_{12}=\frac{Q^{2}}{2 w_{e}}$

$C_{1 n}=\frac{Q^{2}}{w_{e}}$

Donde:

$Q=$ carga de prueba $=\left(5 * 10^{-12}\right) C$

$w_{e}=$ Energía en el campo eléctrico

Mediante la simulación se determina:

$w_{e}=2.196292 * 10^{-12}[J]$. De donde $C_{n}$

$C_{1 n}=\frac{\left(5 * 10^{-12}\right)^{2}}{2.196292 * 10^{-12}}\left[\frac{F}{m}\right]$

$C_{1 \text { simulacion }}=1.138282 * 10^{-11}\left[\frac{F}{m}\right]$ 
Comparación de los resultados teóricos y de simulación de inductancia y capacitancia al neutro. Las siguientes tablas muestran la comparación de la inductancia calculada teóricamente con la obtenida en la simulación

\section{Ecuación analítica Simulación con COMSOL} \begin{tabular}{l|l}
$0.97103 \mathrm{mH} / \mathrm{Km}$ & $0.9650 \mathrm{mH} / \mathrm{Km}$
\end{tabular}

Tabla 1 Comparación del resultado obtenido de inductancia, con la ecuación analítica y la simulación, para la línea monofásica

\section{Ecuación analítica $\quad$ Simulación con COMSOL} \begin{tabular}{l|l}
$1.2080 e-11 \mathrm{~F} / \mathrm{m}$ & $1.216369 e-11 \mathrm{~F} / \mathrm{m}$
\end{tabular}

Tabla 2 Comparación del resultado obtenido de capacitancia al neutro, con la ecuación analítica y la simulación, para la línea monofásica

\begin{tabular}{|c|c|}
\hline Ecuación analítica & Simulación con COMSOL \\
\hline $1.1316 \mathrm{mH} / \mathrm{Km}$ & $1.134 \mathrm{mH} / \mathrm{Km}$ \\
\hline
\end{tabular}

Tabla 3 Resultados de la simulación y teóricos de una línea trifásica con espaciamiento equilátero, inductancia

\section{Ecuación analítica}

Simulación con COMSOL

\begin{tabular}{l|l}
$1.134934 e-11 \mathrm{~F} / \mathrm{m}$ & $1.138282 e-11 \mathrm{~F} / \mathrm{m}$
\end{tabular}

Tabla 4 Resultados de la simulación y teóricos de una línea trifásica con espaciamiento equilátero, capacitancia al neutro

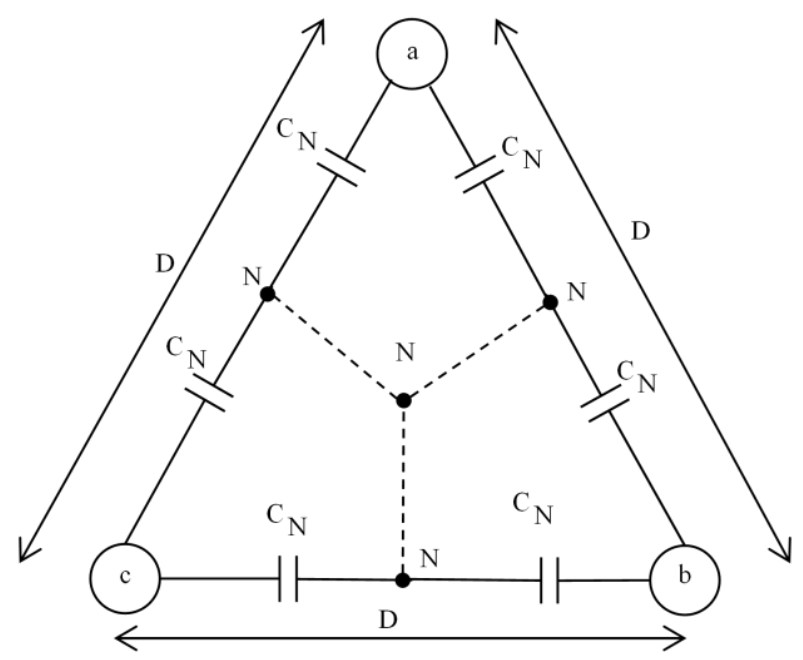

Figura 8 Línea trifásica con espaciamiento equilátero y neutro equidistante de los conductores

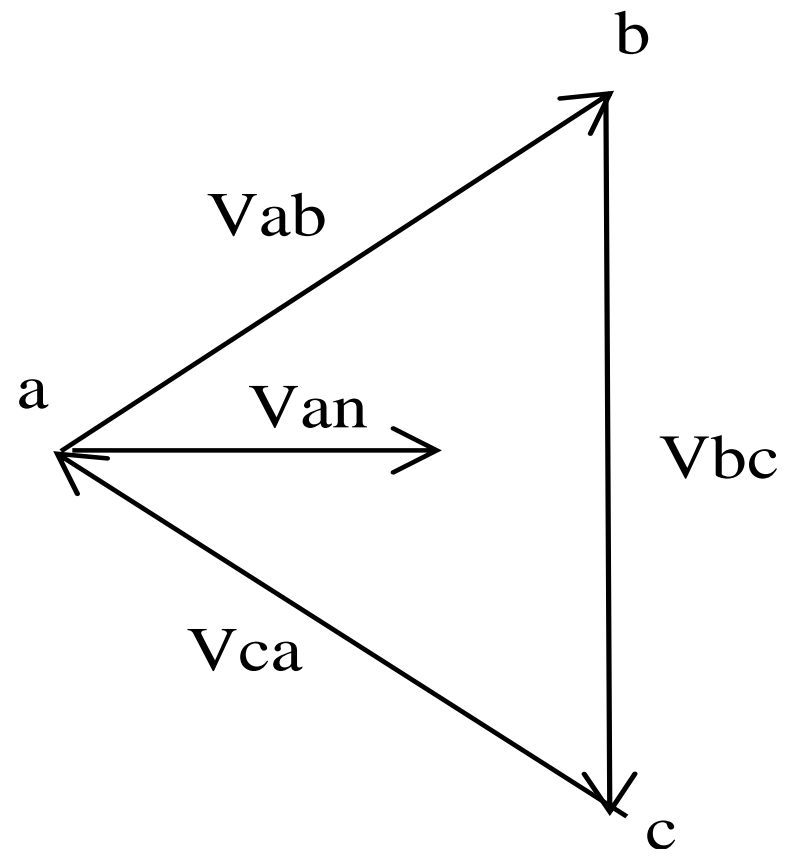

Figura 9 Diagrama fasorial de voltajes balaceados de una línea trifásica

Se puede observar una aceptable concordancia entre los valores obtenidos por medio de las ecuaciones analíticas y la simulación.

\section{Agradecimientos}

Los autores agradecen a la Benemerita Universidad Autonoma de Puebla y a la Universidad de Colima campus Manzanillo por otorgar las facilidades para la presentación de este trabajo.

\section{Conclusiones}

COMSOL Multiphysics es un programa de simulación donde se pueden realizar estudios eléctricos, magnéticos, acústicos, etc. En este caso se utilizó para realizar cálculos de los parámetros (Inductancia y Capacitancia) de Líneas de Transmisión Monofásica y Trifásica en el régimen permanente cuando operan dentro del Sistema Eléctrico, no tomando en cuenta la dispercion lineal asi como el efecto skin. El desarrollo de las ecuaciones analíticas que nos permitieron obtener los parámetros fueron fáciles debido a que la geometría bajo estudio no fue compleja. Así, haciendo uso de COMSOL Multiphysics, que emplea el método de Elemento Finito mediante ecuaciones diferenciales parciales, nos fue posible obtener valores de Capacitancia e Inductancia parecidos a los calculados analíticamente. 
Para concluir, a través del método de elemento finito utilizado por COMSOL Multiphysics es posible verificar la validez de los resultados de las ecuaciones analíticas y entender las restricciones con las cuales se obtienen, en trabajos futuros se trabajará con configuraciones geométricas no equidistantes asi como varios circuitos por torres.

\section{Referencias}

Clayton, P. R. (1994). Analysis of Multiconductor Transmission Lines. washington dc: Wiley.

COMSOL Multiphysics. (2006). COMSOL Multiphysics Manual AC/DC. Obtenido de www.software-

shop.com/in.php?mod=ver_producto\&prdID =2 90 .

COMSOL Multiphysics. (2015). COMSOL Multiphysics. Obtenido de www.softwareshop.com/in.php?mod=ver_producto\&prdID=2 90.

D. Glover, J. S. (2008). Sistemas de Potencia, Análisis y Diseño. Mexico: Thomson.

Grainger, W. J. (1996). Análisis de Sistemas Eléctricos de Potencia. Mexico: Mc Graw Hill.

Gustavsen, B., \& Adam, S. (1988). Combined Phase Domain and Modal Domain Calculation of Transmission Line Transients Based on Vector Fitting. IEEE Trans. Power Delivery, Vol. 13, no. 2, 10.

Morched, Gustavsen, B., \& Tartibi, M. (1999). A Universal Model for Accurate Calculation of Electromagnetic Transients on Overhead Lines and Underground Cables . IEEE Trans. Power Delivery, vol. 14, no. 3, 10.

Moreno, P., Gomez, P., Naredo, J. L., \& J. L. Guardado. (2005). Frequency Domain Transient Analysis of Electrical Networks Including Non-Linear Conditions. Electrical Power and Energy Systems, 15. 